Further Section

Appl Neurophysiol 1978;41:248

\title{
Author Index Vol. 41, 1978
}

Adorjani, C. 134 Amano, K. 66 Andy, O. J. 13

Baba,Y. 188 Balasubramaniam, V. 86 Bertrand, C. 122 Bertrand, R. A. 157 Birg, W. 169 Boëthius,J. 57 Bravo, G. 237

Carlsson, A. M. 57 Colicchio, G. 29

Dieckmann, G. 93

Fukamachi, A. 193

Gentilomo, A. 29 Gildenberg, Ph. L. 113

Haas.H. 134 Hamlin, H. 219 Hardy.J. 157,217 Hawrylyshyn, P. 183

Heimburger, R. F. 43 Herrero, J. 237 Hori, T. 223

Iseki.H. 66 Isobe, I. 193

Kanaka, T. S. 86 Kawabatake, H. 66 Kawamura, H. 66 
Keiler, J. T. 146 Kitamura, K. 66 Klar, M. 169 Krainick, J.-U. 134 Kullberg, G. 79 Kwak, R. 209

Lozano, A. P. 237

Manrique, M. 237 Martinez, S. N. 122 Matsumoto, K. 188 Matsumoto, Y. 188 Mayanagi, Y. 223 Meyer, M. 134 Meyerson, B. A. 57 Milstein, V. 43 Mimura, Y. 188 Miyamoto, T. 188 Miyazaki, M. 193 Molina-Negro, P. 122, Moore, D. 43 Moriyasu, N. 202 Mundinger, F. 169

Nakajima.H. 193 Niizuma,H. 209 Nishimoto, A, 188 Nittner, K. 38,142 Notani, M. 66

Ohi, T. 209 Ohmoto, T. 188 Ohye, C. 193 Okudaira, Y. 209 
Organ,L.W. 183 Otabe, K. 209 Oya,S. 237

Rasmussen, T. 1 Risberg, J. 79 Rossi, G. F. 29

Sakamoto.T. 209 Sano, K. 223 Saso, S. 209 Scerrali, M. 29 Schneider, H. 93

Scholl, H. 142 Schvarcz, J. R. 99 Shibazaki.T. 193 Siegfriedp. 134 Small, I. F. 43 Small, J. G. 43 Suzuki, J. 209

Tanikawa, T. 66 Tasker, R.R. 183 Tew, J. M.jr. 146 Thoden, U. 134

Tsubokawa, T. 202

Vaquero, J. 237 Velamati, S. 13 Voris, H. C. 52

WMsler, W. W. 52 Williams, D. S. 146

Zervas,N.T. 219 Zoll, J. G. 232 